\title{
Structure determination of channel and transport proteins by high-resolution microscopy techniques
}

\author{
Marcel Meury, Daniel Harder, Zöhre Ucurum, \\ Rajendra Boggavarapu, Jean-Marc Jeckelmann \\ and Dimitrios Fotiadis* \\ Institute of Biochemistry and Molecular Medicine, and \\ NCCR TransCure, University of Bern, Bühlstrasse 28, \\ CH-3012 Bern, Switzerland \\ * Corresponding author \\ e-mail: dimitrios.fotiadis@mci.unibe.ch
}

\begin{abstract}
High-resolution microscopy techniques provide a plethora of information on biological structures from the cellular level down to the molecular level. In this review, we present the unique capabilities of transmission electron and atomic force microscopy to assess the structure, oligomeric state, function and dynamics of channel and transport proteins in their native environment, the lipid bilayer. Most importantly, membrane proteins can be visualized in the frozen-hydrated state and in buffer solution by cryo-transmission electron and atomic force microscopy, respectively. We also illustrate the potential of the scintillation proximity assay to study substrate binding of detergent-solubilized transporters prior to crystallization and structural characterization.
\end{abstract}

Keywords: atomic force microscopy; electron crystallography; membrane protein; scintillation proximity assay; transmission electron microscopy; transporter; two-dimensional crystal.

\section{Introduction}

Membrane proteins play a crucial role in many cellular and physiological processes and make up $\sim 30 \%$ of the human proteome (Wallin and von Heijne, 1998; Stevens and Arkin, 2000). Specific mutations in genes encoding membrane proteins are associated with numerous diseases, e.g., congestive heart failure, nephrogenic diabetes insipidus, liver cirrhosis, cataract and cystic fibrosis. Despite their importance for the understanding of molecular cell biology and for the structure-based design of new and highly specific drugs, atomic structures of pro- and eukaryotic membrane proteins are strongly underrepresented in the Protein Data Bank (PDB; http://www.pdb.org). Only around 250 coordinate files of unique membrane protein structures are deposited in the PDB (status: August 2010) compared to the thousands of structures of water-soluble proteins. This low number of PDB files indicates that structure determination of membrane proteins at high-resolution remains a particular challenge for structural biology.

Three methods are currently used to determine the structure of membrane proteins: X-ray crystallography (XRC), cryo-transmission electron microscopy (TEM)/electron crystallography (EC) and nuclear magnetic resonance (NMR) spectroscopy. Most of the membrane protein structures in the PDB were determined using three-dimensional (3D) crystals and XRC. This is a well-established technology that allows atomic structures of proteins to be solved quickly and efficiently. The remaining PDB entries originate from cryoTEM/EC and NMR spectroscopy, to which the former contributed eight atomic models of membrane proteins. This could appear as a modest accomplishment compared to the high number of membrane protein structures solved by XRC. However, considering that less than two dozen groups are currently pursuing electron crystallography vs. hundreds of $\mathrm{X}$-ray groups, the eight membrane protein structures from cryo-TEM/EC compare rather well to those determined by XRC (Hite et al., 2007).

In contrast to XRC and NMR spectroscopy, which are 'allor-nothing' approaches, cryo-TEM/EC of two-dimensional (2D) crystals has been successfully used to determine the projection maps and 3D structures of a large number of membrane proteins at low and medium resolution, i.e., 4-10 (Hite et al., 2007). At these resolutions, valuable information is gained on the arrangement of transmembrane $\alpha$-helices (TMHs) and other secondary structure elements within membrane proteins. Most importantly, reconstitution of membrane proteins into $2 \mathrm{D}$ crystals in the presence of lipids restores the native environment of membrane proteins as well as their biological activity. For example, transporters are unable to transport and certain G-protein-coupled receptors unable to bind their $G$ proteins when solubilized in detergent micelles (Leitz et al., 2006; Whorton et al., 2008). Therefore, the structure of membrane proteins are studied under more native conditions by cryo-TEM/EC of 2D crystals than by XRC and NMR spectroscopy, where detergentsolubilized membrane proteins are almost exclusively used. An excellent paradigm for function (Walz et al., 1994) and structure studies (Murata et al., 2000) of a membrane protein in $2 \mathrm{D}$ crystals is aquaporin-1. Complementary to cryo-TEM/ $\mathrm{EC}$, the topography of reconstituted membrane proteins, i.e., in proteoliposomes, 2D crystals and native membranes, can be recorded by atomic force microscopy (AFM) under nearphysiological conditions and at sub-nanometer resolution. The combined application of these high-resolution microscopy techniques establishes the 3D structure and supramolecular organization of membrane proteins. 
Channel and transport proteins are an important class of membrane proteins, representing at least $10 \%$ of the human genome. They are involved in cellular nutrition, ionic homeostasis and disposal of waste products. In Bern, Switzerland our group focuses on the function and in particular the structure of channel and transport proteins from pro- and eukaryotic organisms using high-resolution microscopy techniques and XRC (Figure 1). This review introduces highresolution microscopy techniques, i.e., TEM and AFM, and summarizes recent results on channel and transport proteins acquired in our laboratory using these techniques.

\section{High-resolution microscopy techniques}

\section{Negative-stain transmission electron microscopy}

The contrast of most biological materials is poor on electron micrographs. This is because electrons are weakly scattered by biological samples, which are mainly composed of elements of low atomic number. To improve contrast, samples are embedded in electron-dense stains, i.e., heavy metal salts, which strongly scatter electrons and thus enhance the contrast. In our laboratory, we routinely use the negative staining technique (Kiselev et al., 1990; Bremer et al., 1998) to study single purified membrane proteins, proteoliposomes, 2D crystals and native membranes (Figure 1). In negative staining, biomolecules are adsorbed on an electron transparent support, embedded in stain and dried. This easy and rapid technique allows the direct visualization of biomolecules by TEM and determination of their molecular shape, size and oligomeric state. Figure 2 summarizes single particle analyses of four channel and transport proteins from our present and past studies. We intentionally chose monomeric, dimeric, trimeric and tetrameric proteins to illustrate the power of negative-stain TEM for the determination of the oligomeric state of membrane proteins. The three transporters in Figure $2 \mathrm{~A}-\mathrm{C}$ show a negative-stain accumulating cavity in each monomer. The oligomeric state of the plant aquaporin SoPIP2;1 (Karlsson et al., 2003) in Figure 2D is less evident, as determined by the electron micrographs and average. However, its square shape suggests that this channel is probably a tetramer. To corroborate the oligomeric state of a membrane protein determined by negative-stain TEM from single particles, we always characterize our target proteins by blue native-polyacrylamide gel electrophoresis and gel filtration, and occasionally also by chemical crosslinking.

Regarding any form of crystallography, the production of well-ordered 2D membrane protein crystals for cryo-TEM/ EC requires extensive screening of numerous conditions. Parameters such as $\mathrm{pH}$, salt, ionic strength, protein concentration, temperature, lipid, lipid-to-protein ratios, etc. have to be varied and then explored for successful 2D crystallization of the proteins. As described nicely by Andreas Engel and colleagues (Jap et al., 1992), 2D crystallization is more an art than a science. For additional general reviews on the 2D crystallization of membrane proteins, see Kühlbrandt (1992) and Hasler et al. (1998).

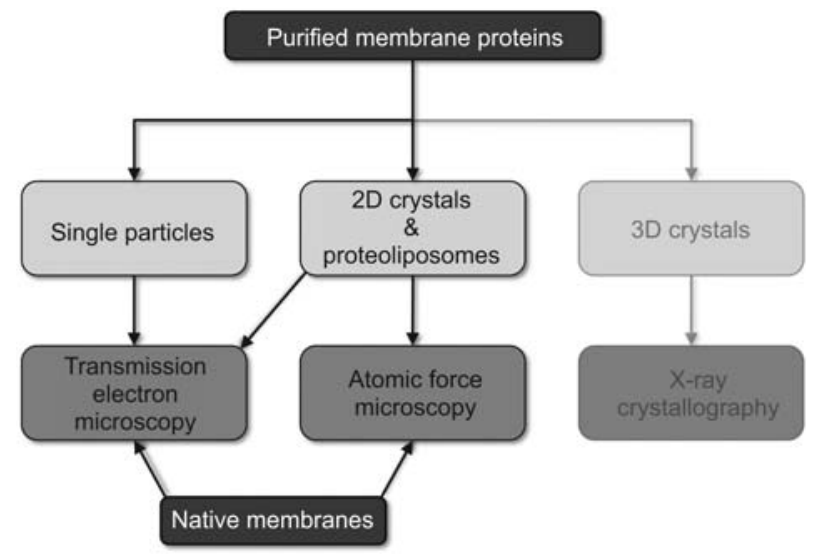

Figure 1 Schematic representation of our working strategies for the determination of the structure and supramolecular organization of channel and transport proteins.

In this review, only high-resolution microscopy techniques, i.e., AFM and TEM, are discussed. XRC of 3D crystals is thus faded in this Figure.

Screening for $2 \mathrm{D}$ crystals is more time-consuming than for $3 \mathrm{D}$ crystals, because for every 2D crystallization condition an electron microscopy grid with the negatively stained specimen has to be prepared and examined by TEM. Negative staining is not only very useful for screening purposes but also to characterize the structure of the crystallized membrane protein at low resolution. During the project 'European Genomics Initiative on Disorders of Plasma

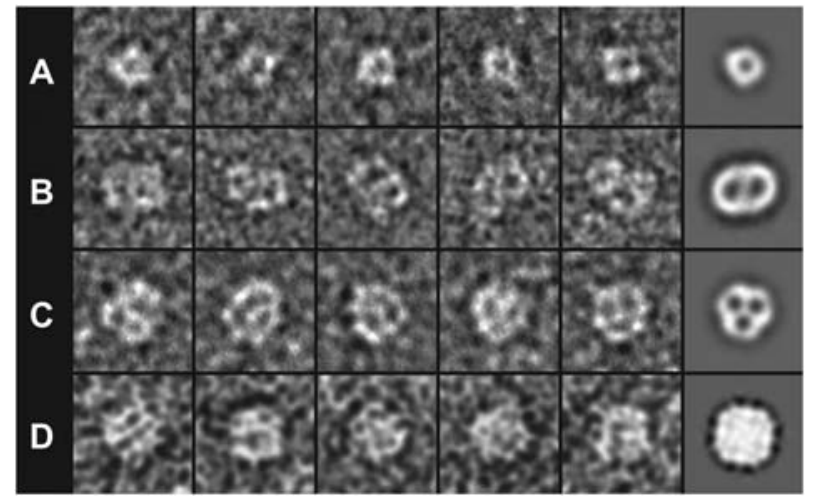

Figure 2 Single particle analysis of purified and negatively stained channel and transport proteins.

(A) The L-serine/L-threonine exchanger SteT (YkbA) from Bacillus subtilis (Reig et al., 2007). SteT is a monomer with a $M_{\mathrm{r}}$ of 49 000. (B) The L-arginine/agmatine antiporter AdiC (YjdE) from Escherichia coli (Casagrande et al., 2008). AdiC is a dimer with a $M_{\mathrm{r}, \mathrm{dimer}}$ of $\sim 97$ 000. (C) The urea transporter dvUT from Desulfovibrio vulgaris (unpublished results). dvUT is a trimer with a $M_{\mathrm{r}, \text { trimer }}$ of $\sim 113$ 000. (D) The plant aquaporin SoPIP2;1 (PM28A) from Spinacia oleracea (Karlsson et al., 2003). SoPIP2;1 forms tetramers with a $M_{\text {r,tetramer }}$ of $\sim 130000$. The first five images (from left to right) in each panel are raw electron micrographs, whereas the sixth rightmost image represents an average of top views of the corresponding protein. The frame size of the images is $198 \AA$. All averages were low-pass filtered at $20 \AA$ resolution. 
Membrane Amino Acid Transporters' (EUGINDAT; 6th European Framework Program), we successfully crystallized a mutant of the L-arginine/agmatine antiporter AdiC from Escherichia coli (AdiC-W293L) in two dimensions. In contrast to wild-type AdiC (wt-AdiC), AdiC-W293L more readily and reproducibly forms well-ordered $2 \mathrm{D}$ crystals. This mutant is unable to bind its substrates, e.g., L-arginine and agmatine, and is thus unable to transport.

Specific point mutants that diminish the number of possible conformations of a transporter and thus foster crystallization were successfully used for the first time for the 3D crystallization of the lactose permease LacY (LacY-C154G) (Abramson et al., 2003). A rapid and sensitive method to assay the substrate binding properties of mutants and of uncharacterized orphan target proteins in general is the scintillation proximity assay (SPA). In 2007, Matthias Quick (Quick and Javitch, 2007) introduced the application of this method to detergent-solubilized membrane transport proteins. We have established the SPA method for detergentsolubilized transporters in Bern, which is now being used routinely. In Figure 3, recent SPA results with wt-AdiC and AdiC-W293L are shown. SPA allows us to measure ligand binding (Figure $3 \mathrm{~A})$, to determine dissociation $\left(K_{\mathrm{d}}\right.$; Figure $3 \mathrm{~B})$ and inhibition constants $\left(K_{\mathrm{i}}\right.$; Figure 3D) and to identify substrate specificities (Figure $3 \mathrm{C}$ ).

A

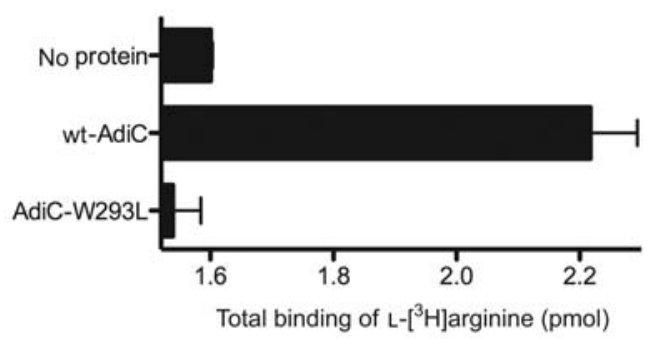

C

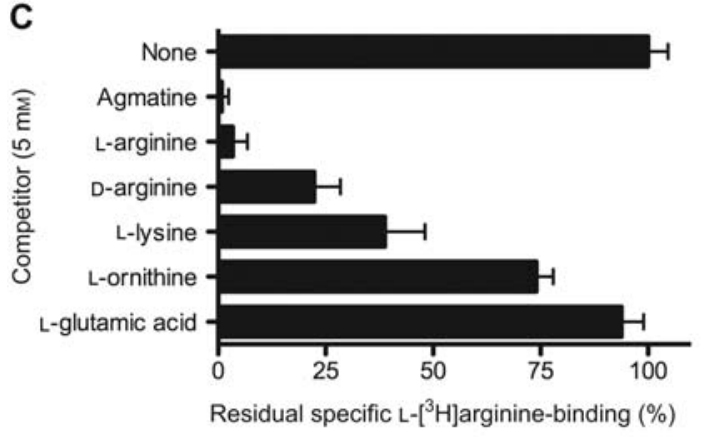

Figure 4A shows an overview electron micrograph of the best 2D crystallization condition for AdiC-W293L and the power spectrum calculated from such a 2D crystal (inset). Sharp diffraction spots can be discerned, indicating the regular order of AdiC-W293L. From such negatively stained 2D crystals (Figure 4A), a projection map can be quickly calculated and structural information gained. Figure $4 \mathrm{~B}$ shows the projection structure (PS) at $28 \AA$ resolution calculated from electron micrographs of negatively stained AdiCW293L crystals. The unit cell is marked and contains four AdiC-W293L dimers. Rows of bright (Figure 4B; white dashed ellipse) and darker (white solid ellipse) AdiC-W293L dimers can be discerned, exhibiting rows exposing either the periplasmic or the cytoplasmic side of the transporter. This suggests that the protein mass protruding out of the membrane is different on the periplasmic and cytoplasmic side of AdiC-W293L.

\section{Cryo-transmission electron microscopy}

Negative-stain TEM does not provide information on the interior of biomolecules, and the maximal resolution is limited to approximately $(13 \AA)^{-1}$ (Darst et al., 1991). The limitations inherent in this method have largely been solved by introducing cryotechniques. In cryo-TEM, 2D crystals are

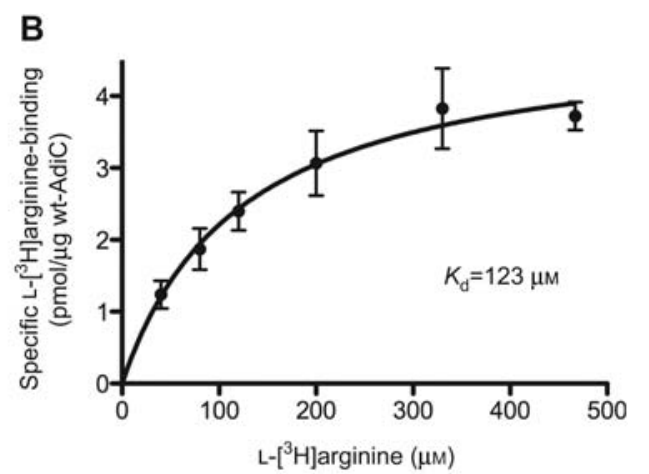

D

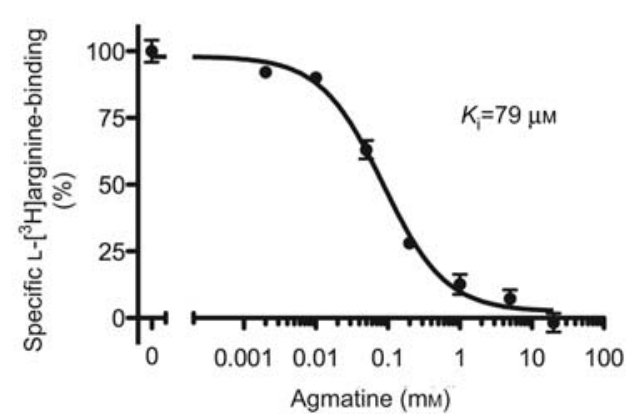

Figure 3 Scintillation proximity assay (SPA) of AdiC.

(A) Wild-type AdiC (wt-AdiC) is able to bind $\mathrm{L}_{-}\left[{ }^{3} \mathrm{H}\right]$ arginine in contrast to the point mutant AdiC-W293L. The background, i.e., binding of the radiotracer to the SPA beads (no protein), is also shown. (B) Saturation experiments of wt-AdiC with increasing concentrations of L- $\left[{ }^{3} \mathrm{H}\right]$ arginine indicate a $K_{\mathrm{d}}$ of $\sim 120 \mu \mathrm{M}$. (C) Inhibition pattern of wt-AdiC. SPA competition experiments were performed with $10 \mu \mathrm{M}$ $\mathrm{L}-\left[{ }^{3} \mathrm{H}\right]$ arginine and $5 \mathrm{~mm}$ of the corresponding substrate analog. The sample without competitor (none) represents $100 \% \mathrm{~L}-\left[{ }^{3} \mathrm{H}\right] \mathrm{arginine}$ binding to wt-AdiC. (D) Competitive inhibition of $\mathrm{L}-\left[{ }^{3} \mathrm{H}\right]$ arginine $(10 \mu \mathrm{M})$ binding to wt-AdiC with increasing concentrations of agmatine. Bars and data points in (A)-(D) represent mean \pm SEM from at least triplicate determinations. All SPAs were conducted according to Quick and Javitch (2007) using a TopCount scintillation counter (Perkin Elmer, Waltham, MA, USA). 


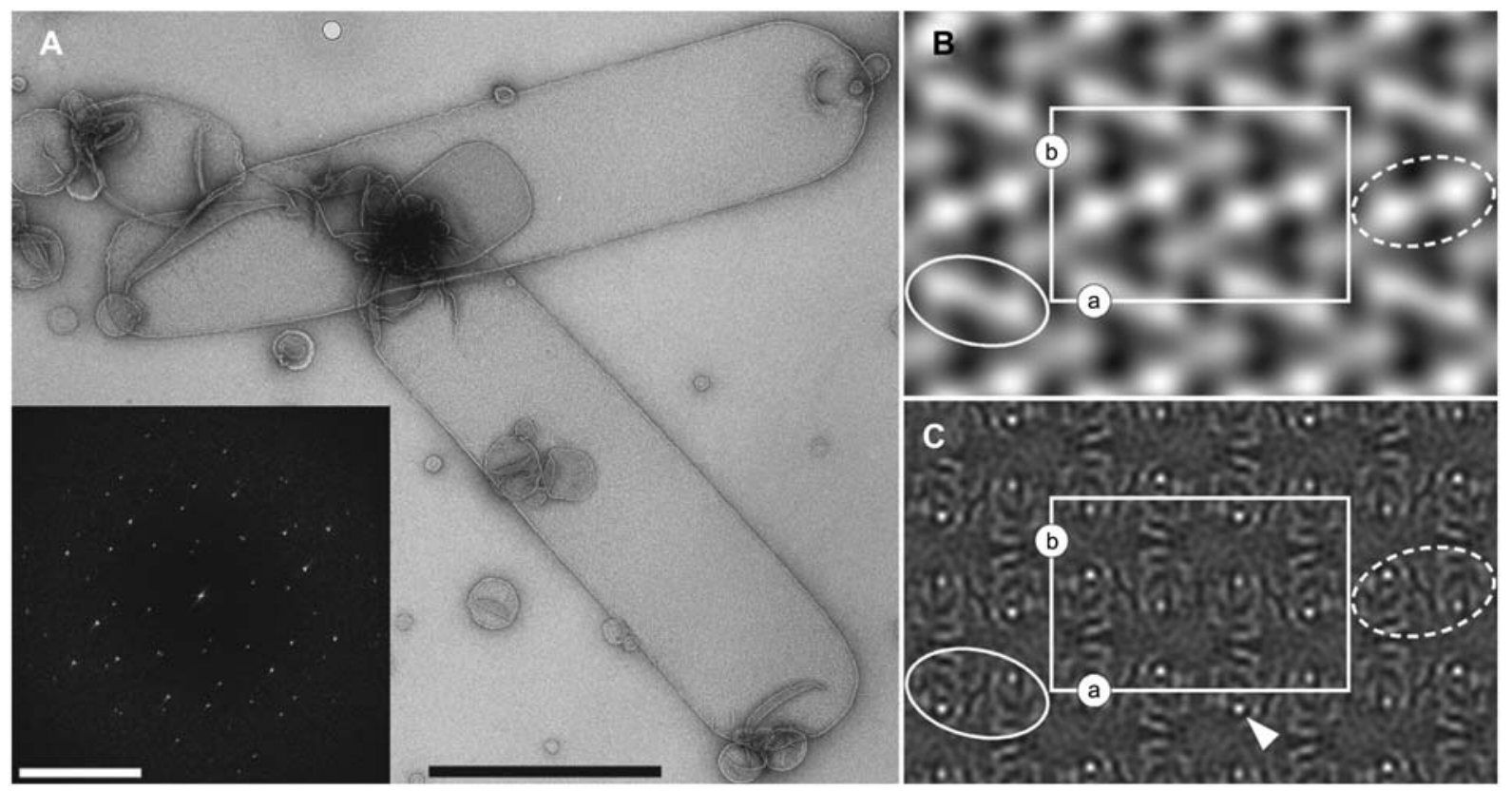

Figure 4 Transmission electron microscopy of 2D crystals of the L-arginine/agmatine antiporter AdiC-W293L.

(A) Overview micrograph of negatively stained AdiC-W293L 2D crystals adsorbed on carbon film. The crystals have a tube-like appearance. The inset shows a typical power spectrum calculated from a negatively stained 2D AdiC-W293L crystal. Diffraction spots can be discerned, indicating crystallinity. Projection maps calculated from negatively stained (B) and unstained, trehalose-embedded AdiC-W293L 2D crystals (C; imposed $p 222_{1} 2_{1}$ symmetry) (Casagrande et al., 2008). The white rectangle marks the unit cell (lattice parameters: $\mathrm{a}=184 \AA$, $b=119 \AA$, $\gamma=90^{\circ}$, which contains four AdiC-W293L dimers, two up- and two down-oriented. In negative-stain, differently oriented dimers appear brighter or darker and are marked by dashed and solid white ellipses. The scale bars represent $1 \mu \mathrm{m}(\mathrm{A})$ and $(25 \AA)^{-1}$ (inset in A). In (B) and $(\mathrm{C})$, protein density appears white.

not stained but embedded in vitreous ice or sugars, e.g., glucose and trehalose, and imaged at cryogenic temperatures. Thus, biomolecules are neither fixed nor dried, and are studied in their native and hydrated state.

Transmission electron microscopes equipped with a fieldemission gun transfer structural information from thin samples at the atomic scale. Electron micrographs represent a projection of the 3D Coulomb potential distribution of the imaged object. For structure determination of membrane proteins by cryo-TEM, images from non-tilted and tilted 2D crystals are recorded, and the structural information is then extracted from electron micrographs by applying computer image processing. Typical software are the $2 \mathrm{dx}$ (Gipson et al., 2007) and the Image Processing Library \& Toolbox (IPLT) (Philippsen et al., 2003, 2007) systems, and the Medical Research Council (MRC) programs (Crowther et al., 1996). In contrast to $\mathrm{XRC}, 3 \mathrm{D}$ structure determination at high resolution by cryo-TEM/EC is still a tedious and time-consuming task, although the methodology has made major advances over the past years. The best example of this progress is reflected in the structure of mammalian AQP0 at $1.9 \AA$ resolution, which was solved by electron diffraction of 2D crystals and molecular replacement (Gonen et al., 2005). This high-resolution structure demonstrates the great potential of EC to solve the structure of membrane proteins and, importantly, to unravel their interactions with individual lipid molecules of the lipid bilayer.
The PS of a membrane protein from 2D crystals is determined quickly. Figure 4C shows the projection map of AdiCW293L at $6.5 \AA$ resolution (Casagrande et al., 2008). The elongated densities (in white) within monomers suggest that the fold of AdiC consists of mainly tilted TMHs, with the exception of one single strong density peak (arrowhead). The latter would suggest a TMH parallel to the membrane normal, when considering the PS of the light-driven proton pump bacteriorhodopsin, which consists of seven perpendicular membrane-embedded TMHs (Grigorieff et al., 1996). Recently, the atomic structure of AdiC was solved by XRC of 3D crystals (Fang et al., 2009; Gao et al., 2009, 2010). These 3D structures elucidated important aspects of the transport mechanism of AdiC and confirmed that the fold of this transporter consists of tilted TMHs. Additionally, they indicated that the strong density peak in the cryo-TEM PS arises from two overlapping tilted TMHs instead of one perpendicular TMH. The existence of 3D AdiC structures allowed their comparison with the PS of AdiC-W293L. The former were determined by XRC of 3D crystals of detergentsolubilized wt-AdiC, whereas the latter was determined by cryo-TEM of 2D crystals of lipid-embedded AdiC-W293L.

In contrast to membrane proteins in detergent micelles, membrane proteins in the lipid bilayer experience a lateral pressure of $\sim 30 \mathrm{mN} \mathrm{m}^{-1}$ (Rosenbusch, 2001). This could result in structures of the same protein in different conformational states and could be particularly expected for trans- 
porters, because these proteins adopt different conformational states during substrate translocation, e.g., occluded, inward- and outward-facing conformations, and are thus structurally flexible. To make such a comparison possible, we elaborated a method to calculate and visualize PSs from PDB coordinate files: the Projection Structure Visualization (PSV) method (Jeckelmann et al., 2010). The 3D structure of wt-AdiC was solved in the outward-facing substrate-free state. Comparison of its PSV method calculated PS with the cryo-TEM PS of membrane-embedded AdiC-W293L revealed distinct structural differences (Figure 5). These findings are of particular interest because they can reflect a different conformation of AdiC, e.g., the inward-facing conformation, in the transport cycle, induced by the lateral pressure exerted on the AdiC protein when embedded in the lipid bilayer (see Figure 5 legend for a detailed description of the observed differences).

\section{Atomic force microscopy (AFM)}

The principle of AFM is simple and best compared to that of a record player, where a needle moves over ridges and valleys in the groove of a record. In AFM, a nanometer-sized sharp tip mounted on a cantilever spring (typically 100$400 \mu \mathrm{m}$ long) is raster scanned over the sample surface. The topography of the sample is reconstructed from the up-anddown displacements of the cantilever arm recorded as the tip is scanned.

During more than two decades of continuous technological and methodological development, AFM has become a powerful tool for imaging the surface structure of biomolecules. Of particular interest for biologists is the possibility to observe biological samples under physiologically relevant conditions, i.e., in buffer solution, at normal pressure and at temperatures between $4^{\circ} \mathrm{C}$ and $37^{\circ} \mathrm{C}$. Because biomolecules are 'alive' under these native conditions, AFM has been successfully used to observe single proteins at work; for reviews, see Engel et al. (1999) and Engel and Müller (2000). One of the most exciting examples in the literature for the direct visualization of conformational changes is the $\mathrm{pH}$ and $\mathrm{Ca}^{2+}$ dependent opening and closing of the gap junction channel connexin26 (Müller et al., 2002; Yu et al., 2007). Because of these unique capabilities, AFM is currently being used as a complementary technique to XRC and electron microscopy.

Another important feature of this instrument is the exceptionally high signal-to-noise ratio of AFM topographs (see Figure 6 for examples). On such micrographs submolecular details of single proteins are resolved to a maximal lateral resolution of $\sim 5 \AA$ and a vertical resolution of $\sim 1 \AA$. Nevertheless, high resolutions are only achieved on reconstituted membrane protein surfaces, i.e., densely packed proteoliposomes, 2D crystals and native membranes, immobilized on mica using contact mode AFM. In this mode, samples are raster scanned at constant forces between 50 and $100 \mathrm{pN}$ thus minimizing possible damage of the soft biological specimen. By contrast, application of higher forces can be advantageous in order to mechanically dissect biological samples using the AFM stylus as a nanoscalpel (Fotiadis et al., 1998,
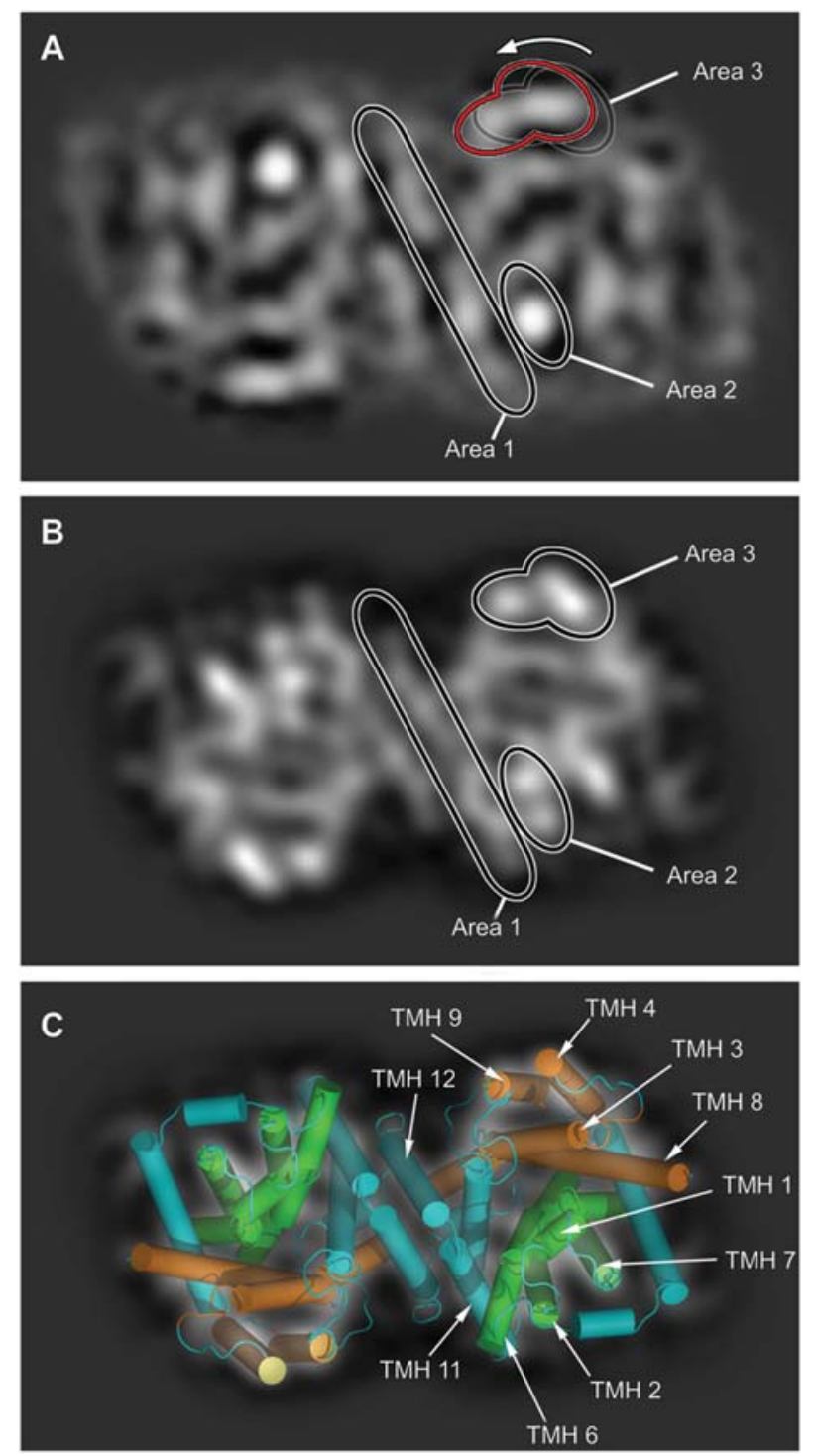

Figure 5 Comparison of PSs of substrate-free AdiCs embedded in lipid bilayers (A) and in detergent micelles (B and C).

(A) PS of AdiC-W293L dimer at $6.5 \AA$ resolution determined by cryo-TEM of 2D crystals (Casagrande et al., 2008). (B) PS of wtAdiC at $6.0 \AA$ resolution (PDB code 3LRB) (Gao et al., 2009) calculated by the PSV method (Jeckelmann et al., 2010). In panels (A) and (B), Area 1 is composed of the dimerization TMHs 11 and 12. The position and shape of these helices are well-conserved between (A) and (B). The strong density in Area 2 in panel (A) is split into two maxima in panel (B), indicating a different arrangement of the 'rocking bundle' domain. In addition, the positions of the densities in Area 3, which are part of the 'hash' domain, differ between the panels and seem to be pushed counterclockwise towards the dimerization interface (see arrow and red contour line) in the cryo-TEM PS (A). (C) Superposition of the PSV method calculated PS with the corresponding X-ray structure shown as a cartoon representation. TMHs are colored in cyan except for the 'rocking bundle' domain (green; composed of TMHs 1, 2, 6 and 7) and the 'hash' domain (orange; composed of TMHs 3, 4, 8 and 9). The 'rocking bundle' and 'hash' domains are thought to move during substrate translocation (Forrest et al., 2008; Shimamura et al., 2010). The frame size of the images is $125 \times 75 \AA$. 


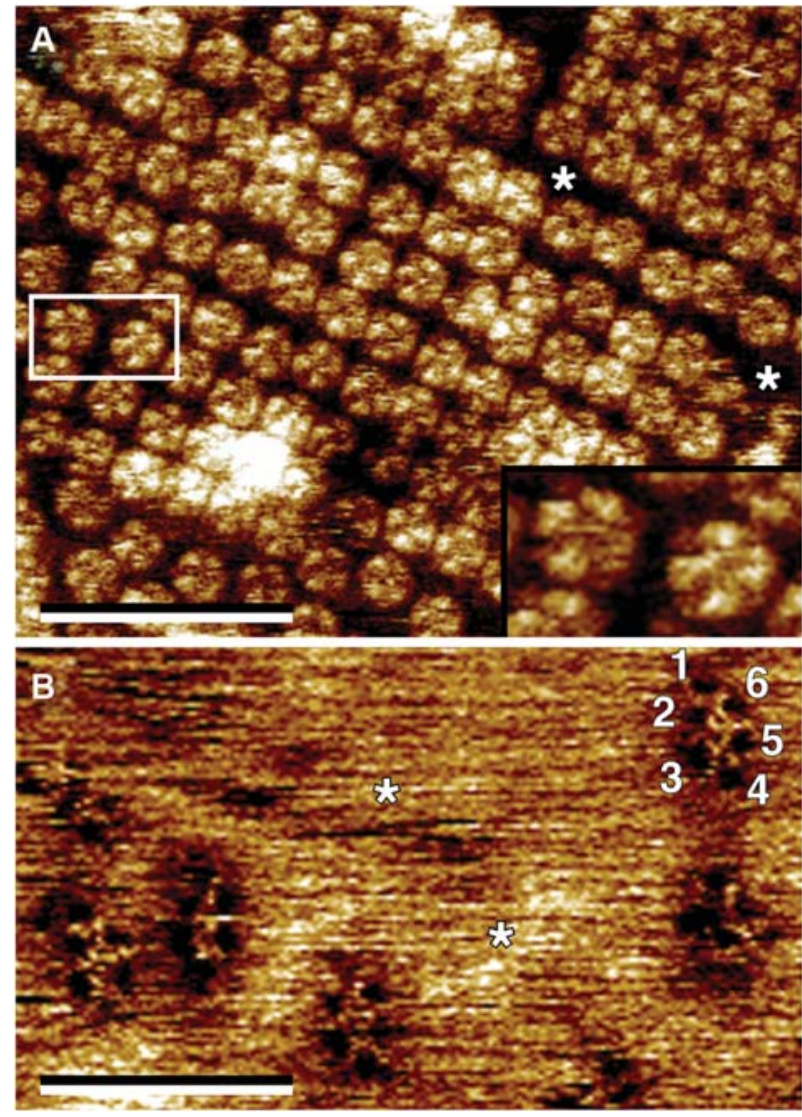

Figure 6 High-resolution AFM topographs of aquaporin-0 (AQP0, MIP) (A) (Fotiadis et al., 2000) and the voltage-dependent anion channel (VDAC) (B) (Hoogenboom et al., 2007).

(A) In lipid bilayers reconstituted ovine AQP0 forms 2D crystals (top right) and densely packed regions. Single AQP0 tetramers are clearly visible (extracellular side of AQP0). The inset shows a magnification of the two AQP0 tetramers marked by the rectangular box. AQP0 protein mass, e.g., loop domains, protrudes $\sim 15 \AA$ out of the lipid bilayer (asterisks). (B) Holes formed by VDAC proteins can be discerned in the native outer mitochondrial membrane. VDAC adopts different oligomeric states. The characteristic six pores (labeled 1-6) of VDAC hexagons (hexamers) are recognizable. In contrast to AQP0, almost no protein mass protrudes out of the lipid bilayer (asterisks) on the intermembrane space side of the VDAC. Both AFM images were recorded in contact mode, at nearphysiological conditions, i.e., in buffer solution, and at room temperature and normal pressure. The resolution of the topographs is better than $10 \AA$. The vertical brightness ranges are $16 \AA(\mathrm{A})$ and $4 \AA$ (B). The frame size of the inset in panel (A) is $175 \times 98 \AA$. Scale bars represent $300 \mathrm{~nm}$.

2000, 2002). High-resolution AFM topographs, as shown in Figure 6, are acquired line-by-line at typical scan rates of 4-10 lines/s. Therefore, the acquisition of one AFM topograph usually takes one to a few minutes. Such time resolutions only allow slow dynamics and conformational changes of proteins to be captured. Recently, high-speed AFM has been introduced making the visualization of dynamic biomolecular processes possible at millisecond and nanometer resolution (Humphris et al., 2005; Ando et al., 2008).

\section{Conclusion and future prospects}

High-resolution microscopy techniques afford great opportunities to assess the structure, oligomeric state, function and dynamics of channel and transport proteins, and of membrane proteins in general. The main bottleneck for successful high-resolution structure determination by cryoTEM/EC is the same as for NMR spectroscopy and XRC: the production and purification of milligram amounts of stable and healthy membrane proteins. Such proteins are then studied by NMR spectroscopy in their detergent-solubilized form or crystallized in two- and three-dimensions for cryoTEM/EC and XRC. Crystallization of membrane proteins and especially transport proteins represents an additional bottleneck, because transporters can adopt different conformational states, e.g., occluded, inward- and outward-facing conformations, introducing structural heterogeneities.

In contrast to $3 \mathrm{D}$ crystals, membrane proteins assembled in $2 \mathrm{D}$ crystals are embedded in lipid bilayers, where the lateral pressure, and thus also their native environment, is reestablished. With highly ordered 2D membrane protein crystals, cryo-TEM/EC provides information on their projection and 3D structure down to $1.9 \AA$ resolution. With the aid of the PSV method, we have shown that conformational changes and structural differences are also detected in projection. The surface structures and dynamics of single membrane proteins at work are revealed by AFM at subnanometer resolution and under near-physiological conditions. Because of the high signal-to-noise ratio of AFM topographs, well-ordered 2D crystals are not a prerequisite for such studies, and densely packed proteoliposomes completely suffice, thus facilitating the work significantly. The number of laboratories using such high-resolution microscopy techniques compared to those using XRC is low but increasing.

In this article, we have focused on the high-resolution aspects of electron and atomic force microscopy. However, it should also be noted that these techniques offer a unique advantage for the multiresolution visualization of biological structures from the cellular level down to the molecular level. Although efforts are needed for further development of these microscopy-based methods, particularly in automated image/ data acquisition, processing and analysis, these techniques show great potential to innovate cell and molecular biology in the near future.

\section{Acknowledgments}

The authors thank Leah Witton for reading the manuscript. This research was supported by the Swiss National Foundation for Scientific Research (Grant 31003A_125150), the Bern University Research Foundation, the Novartis Foundation, the European Science Foundation (Grant 09-EuroSYNBIO-FP-012 NANOCELL) and the National Center of Competence in Research (NCCR) TransCure. 


\section{References}

Abramson, J., Smirnova, I., Kasho, V., Verner, G., Kaback, H.R., and Iwata, S. (2003). Structure and mechanism of the lactose permease of Escherichia coli. Science 301, 610-615.

Ando, T., Uchihashi, T., Kodera, N., Yamamoto, D., Miyagi, A., Taniguchi, M., and Yamashita, H. (2008). High-speed AFM and nano-visualization of biomolecular processes. Pflüger's Arch. 456, 211-225.

Bremer, A., Häner, M., and Aebi, U. (1998). Negative staining. In: Cell Biology: A Laboratory Handbook, 2nd Edn., Vol. 3, J.E. Celis, ed. (San Diego, CA: Academic Press, Inc.), pp. 277-284.

Casagrande, F., Ratera, M., Schenk, A.D., Chami, M., Valencia, E., Lopez, J.M., Torrents, D., Engel, A., Palacin, M., and Fotiadis, D. (2008). Projection structure of a member of the amino acid/ polyamine/organocation transporter superfamily. J. Biol. Chem. 283, 33240-33248.

Crowther, R.A., Henderson, R., and Smith, J.M. (1996). MRC image processing programs. J. Struct. Biol. 116, 9-16.

Darst, S.A., Kubalek, E.W., Edwards, A.M., and Kornberg, R.D. (1991). Two-dimensional and epitaxial crystallization of a mutant form of yeast RNA polymerase II. J. Mol. Biol. 221, 347-357.

Engel, A. and Müller, D.J. (2000). Observing single biomolecules at work with the atomic force microscope. Nat. Struct. Biol. 7, 715-718.

Engel, A., Lyubchenko, Y., and Müller, D.J. (1999). Atomic force microscopy: a powerful tool to observe biomolecules at work. Trends Cell Biol. 9, 77-80.

Fang, Y., Jayaram, H., Shane, T., Kolmakova-Partensky, L., Wu, F., Williams, C., Xiong, Y., and Miller, C. (2009). Structure of a prokaryotic virtual proton pump at $3.2 \AA$ resolution. Nature 460 , 1040-1043.

Forrest, L.R., Zhang, Y.W., Jacobs, M.T., Gesmonde, J., Xie, L., Honig, B.H., and Rudnick, G. (2008). Mechanism for alternating access in neurotransmitter transporters. Proc. Natl. Acad. Sci. USA $105,10338-10343$.

Fotiadis, D., Müller, D.J., Tsiotis, G., Hasler, L., Tittmann, P., Mini, T., Jeno, P., Gross, H., and Engel, A. (1998). Surface analysis of the photosystem I complex by electron and atomic force microscopy. J. Mol. Biol. 283, 83-94.

Fotiadis, D., Hasler, L., Müller, D.J., Stahlberg, H., Kistler, J., and Engel, A. (2000). Surface tongue-and-groove contours on lens MIP facilitate cell-to-cell adherence. J. Mol. Biol. 300, 779-789.

Fotiadis, D., Scheuring, S., Müller, S.A., Engel, A., and Müller, D.J. (2002). Imaging and manipulation of biological structures with the AFM. Micron 33, 385-397.

Gao, X., Lu, F., Zhou, L., Dang, S., Sun, L., Li, X., Wang, J., and Shi, Y. (2009). Structure and mechanism of an amino acid antiporter. Science 324, 1565-1568.

Gao, X., Zhou, L., Jiao, X., Lu, F., Yan, C., Zeng, X., Wang, J., and Shi, Y. (2010). Mechanism of substrate recognition and transport by an amino acid antiporter. Nature 463, 828-832.

Gipson, B., Zeng, X., Zhang, Z.Y., and Stahlberg, H. (2007). 2dx user-friendly image processing for 2D crystals. J. Struct. Biol. 157, 64-72.

Gonen, T., Cheng, Y., Sliz, P., Hiroaki, Y., Fujiyoshi, Y., Harrison, S.C., and Walz, T. (2005). Lipid-protein interactions in doublelayered two-dimensional AQP0 crystals. Nature 438, 633-638.

Grigorieff, N., Ceska, T.A., Downing, K.H., Baldwin, J.M., and Henderson, R. (1996). Electron-crystallographic refinement of the structure of bacteriorhodopsin. J. Mol. Biol. 259, 393-421.
Hasler, L., Heymann, J.B., Engel, A., Kistler, J., and Walz, T. (1998). 2D crystallization of membrane proteins: rationales and examples. J. Struct. Biol. 121, 162-171.

Hite, R.K., Raunser, S., and Walz, T. (2007). Revival of electron crystallography. Curr. Opin. Struct. Biol. 17, 389-395.

Hoogenboom, B.W., Suda, K., Engel, A., and Fotiadis, D. (2007). The supramolecular assemblies of voltage-dependent anion channels in the native membrane. J. Mol. Biol. 370, 246-255.

Humphris, A.D.L., Miles, M.J., and Hobbs, J.K. (2005). A mechanical microscope: high-speed atomic force microscopy. Appl. Phys. Lett. 86, 034106.

Jap, B.K., Zulauf, M., Scheybani, T., Hefti, A., Baumeister, W., Aebi, U., and Engel, A. (1992). 2D crystallization: from art to science. Ultramicroscopy 46, 45-84.

Jeckelmann, J.-M., Palacin, M., and Fotiadis, D. (2011). A tool for the qualitative comparison of membrane-embedded and detergent-solubilized membrane protein structures in projection. J. Struct. Biol. in press.

Karlsson, M., Fotiadis, D., Sjovall, S., Johansson, I., Hedfalk, K., Engel, A., and Kjellbom, P. (2003). Reconstitution of water channel function of an aquaporin overexpressed and purified from Pichia pastoris. FEBS Lett. 537, 68-72.

Kiselev, N.A., Sherman, M.B., and Tsuprun, V.L. (1990). Negative staining of proteins. Electron Microsc. Rev. 3, 43-72.

Kühlbrandt, W. (1992). Two-dimensional crystallization of membrane proteins. Q. Rev. Biophys. 25, 1-49.

Leitz, A.J., Bayburt, T.H., Barnakov, A.N., Springer, B.A., and Sligar, S.G. (2006). Functional reconstitution of $\beta 2$-adrenergic receptors utilizing self-assembling nanodisc technology. Biotechniques 40, 601-610.

Müller, D.J., Hand, G.M., Engel, A., and Sosinsky, G. (2002). Conformational changes in surface structures of isolated Connexin 26 gap junctions. EMBO J. 21, 3598-3607.

Murata, K., Mitsuoka, K., Hirai, T., Walz, T., Agre, P., Heymann, J.B., Engel, A., and Fujiyoshi, Y. (2000). Structural determinants of water permeation through aquaporin-1. Nature 407, 599-605.

Philippsen, A., Schenk, A.D., Stahlberg, H., and Engel, A. (2003). Iplt - image processing library and toolkit for the electron microscopy community. J. Struct. Biol. 144, 4-12.

Philippsen, A., Schenk, A.D., Signorell, G.A., Mariani, V., Berneche, S., and Engel, A. (2007). Collaborative EM image processing with the IPLT image processing library and toolbox. J. Struct. Biol. 157, 28-37.

Quick, M. and Javitch, J.A. (2007). Monitoring the function of membrane transport proteins in detergent-solubilized form. Proc. Natl. Acad. Sci. USA 104, 3603-3608.

Reig, N., del Rio, C., Casagrande, F., Ratera, M., Gelpi, J.L., Torrents, D., Henderson, P.J., Xie, H., Baldwin, S.A., Zorzano, A., et al. (2007). Functional and structural characterization of the first prokaryotic member of the L-amino acid transporter (LAT) family: a model for APC transporters. J. Biol. Chem. 282, 13270-13281.

Rosenbusch, J.P. (2001). Stability of membrane proteins: relevance for the selection of appropriate methods for high-resolution structure determinations. J. Struct. Biol. 136, 144-157.

Shimamura, T., Weyand, S., Beckstein, O., Rutherford, N.G., Hadden, J.M., Sharples, D., Sansom, M.S., Iwata, S., Henderson, P.J., and Cameron, A.D. (2010). Molecular basis of alternating access membrane transport by the sodium-hydantoin transporter Mhp1. Science 328, 470-473.

Stevens, T.J. and Arkin, I.T. (2000). Do more complex organisms have a greater proportion of membrane proteins in their genomes? Proteins 39, 417-420. 
Wallin, E. and von Heijne, G. (1998). Genome-wide analysis of integral membrane proteins from eubacterial, archaean, and eukaryotic organisms. Protein Sci. 7, 1029-1038.

Walz, T., Smith, B.L., Zeidel, M.L., Engel, A., and Agre, P. (1994). Biologically active two-dimensional crystals of aquaporin CHIP. J. Biol. Chem. 269, 1583-1586.

Whorton, M.R., Jastrzebska, B., Park, P.S., Fotiadis, D., Engel, A., Palczewski, K., and Sunahara, R.K. (2008). Efficient coupling of transducin to monomeric rhodopsin in a phospholipid bilayer. J. Biol. Chem. 283, 4387-4394.

Yu, J., Bippes, C.A., Hand, G.M., Müller, D.J., and Sosinsky, G.E. (2007). Aminosulfonate modulated $\mathrm{pH}$-induced conformational changes in connexin26 hemichannels. J. Biol. Chem. 282, 8895-8904.

Received August 31, 2010; accepted September 16, 2010 